\title{
The effect of alcohol dose on the development of hypertension in Asian and Western men: systematic review and meta-analysis
}

\author{
Mi-Hyang Jung ${ }^{1,},{ }^{\star}$, Ein-Soon Shin ${ }^{2,}$, , Sang-Hyun $\mathrm{Ihm}^{3}$, Jin-Gyu Jung ${ }^{4}$, Hae-Young Lee ${ }^{5}$, and \\ Cheol-Ho Kim ${ }^{6}$
}

\author{
${ }^{1}$ Cardiovascular Center, Hallym \\ University Chuncheon Sacred \\ Heart Hospital, Chuncheon; \\ ${ }^{2}$ Research Agency for Clinical \\ Practice Guidelines, KAMS Research \\ Center, Korean Academy of Medical \\ Sciences, Seoul; ${ }^{3}$ Division of \\ Cardiology, Department of Internal \\ Medicine, College of Medicine, The \\ Catholic University of Korea, Seoul; \\ ${ }^{4}$ Department of Family Medicine, \\ Research Institute for Medical \\ Sciences, Chungnam National \\ University College of Medicine, \\ Daejeon; ${ }^{5}$ Department of Internal \\ Medicine, Seoul National University \\ Hospital, Seoul; ${ }^{6}$ Department of \\ Internal Medicine, Seoul National \\ University Bundang Hospital, \\ Seongnam, Korea
}

Background/Aims: There are inconsistencies in the effects of low to moderate dose alcohol consumption on the development of hypertension in adult men. We hypothesized that a region-specific effect might participate in this heterogeneity.

Methods: We conducted a systematic review and meta-analysis to evaluate the effect of alcohol dose on hypertension incidence using contemporary data through December 2017. Subjects were categorized according to their level of alcohol consumption as non-drinkers (reference) and low- (0.01 to $20.0 \mathrm{~g} /$ day), moderate- (20.1 to 40.0 g/day), moderate- to high- ( 40.1 to $60.0 \mathrm{~g} /$ day), and high-dose (> 60.0 g/day) drinkers. We defined hypertension as a blood pressure $\geq 140 / 90 \mathrm{mmHg}$ and/or the use of anti-hypertensive drugs.

Results: In total, 11 articles (seven Asian and four Western) were selected for our analysis. Among Asian men, a significantly elevated risk was observed even in the low alcohol dose group in comparison with the group with no alcohol consumption, and the risk increased in a dose-dependent manner (pooled relative risks [95\% confidence intervals (CI)]: 1.25 [1.13 to 1.38], 1.48 [1.27 to 1.72], 1.75 [1.43 to 2.15], and 1.78 [1.51 to 2.09]). Among Western men, a similar dose-response relationship was noted in general ( $p$ for subgroup difference $>0.1$ ), but a significantly elevated risk was evident only in the high-dose group (pooled relative risks [95\% CI]: 1.22 [0.85 to 1.74], 1.57 [0.90 to 2.75 ], 1.47 [0.44 to 4.91 ], and 1.49 [1.02 to 2.18 ]).

Conclusions: Even low doses of alcohol can lead to the development of hypertension, particularly in Asian men. Our findings could serve as additional evidence for developing an appropriate preventive strategy in each region.

Keywords: Alcohol; Hypertension; Ethnic groups; Dose-response relationship; Meta-analysis

\footnotetext{
Correspondence to Sang-Hyun Ihm, M.D.

Division of Cardiology, Department of Internal Medicine, College of Medicine, Bucheon St. Mary's Hospital, The Catholic University of Korea, 327 Sosa-ro, Wonmi-gu, Bucheon 14647, Korea

Tel: +82-32-340-7027, Fax: +82-32-340-2669, E-mail: heartihmsh@yahoo.co.kr https://orcid.org/oooo-0001-5017-5421

"These authors contributed equally to this work.

${ }^{\dagger}$ Current affiliation: Cardiovascular Center, Hallym University Dongtan Sacred Heart Hospital, Hwaseong, Korea
}

Received: January 10, 2019
Revised : February 7, 2019

Accepted: February 8, 2019 


\section{INTRODUCTION}

Hypertension is highly prevalent and corresponds to an important disease burden worldwide [1]. It is important to identify and control the potential risk factors for hypertension to reduce the disease burden. Alcohol is a preventable risk factor for hypertension [2-4]. Despite its clinical relevance, alcohol-related counselling is not adequately provided in the real world [5]. One of the reasons for insufficient counselling is that there have been inconclusive data regarding the effect of low to moderate doses of alcohol on the development of hypertension [6-10]. This uncertainty is partly due to diverse clinical settings, including different ethnicities and sex, which results in heterogeneity in body size, body composition, and alcohol metabolism [6,10-12]. Therefore, the sexand region (ethnicity)-specific effects should be considered in evaluating the effect of the alcohol dose on the incidence of hypertension.

Most guidelines recommended limiting alcohol consumption to less than 2 units/day for men and less than 1 unit/day for women to control blood pressure $[3,4]$. However, those guidelines are based on Western data, and it is not clear whether the same strategy can be directly applied to the Asian population. In the current study, we confined our focus to the dose-response relationship in men. In most studies, especially from Asian countries, the amount of alcohol consumed by women is not high enough to draw any significant dose-response relationships $[13,14]$. More importantly, the disease burden of alcohol-related injury in women is relatively lower than that in men and was less frequently associated with high-risk alcohol consumption features [15-18]. Although some meta-analyses have evaluated the dose-response relationship between alcohol consumption and hypertension incidence [19-21], those studies did not primarily evaluate a region-specific effect. More finely tuned convincing data reflecting local and regional characteristics are warranted to establish effective preventive strategies. From this perspective, we performed a systematic review and meta-analysis to clarify the region-varying effect of alcohol dose on hypertension incidence in adult men using contemporary data through December 2017.

\section{METHODS}

\section{Search strategy}

We searched the PubMed, EMBASE, Cochrane Central Register of Controlled Trials (CENTRAL), PsycINFO, Western Pacific Regional Index Medicus, and domestic databases including RISS, KoreaMed, Kmbase, and NDSL for relevant articles published up to December 2017. Manual searching of the references of two previously published systematic reviews and meta-analyses was also performed $[19,20]$. The detailed search strategy is provided in Supplementary Table 1.

\section{Study selection}

Covidence Software, a standard production platform for Cochrane Reviews, was used for screening (https:// www/covidence.org/). Two independent authors (E.S.S. and S.H.I.) screened the titles and abstracts of the studies identified from the search to eliminate studies that were not relevant to this review. When the title and abstract did not have sufficient information to be screened properly, we retrieved a full-text copy to review. We included studies that met the following criteria:(1) prospective cohort studies and case-control studies on the incidence of hypertension, (2) the exposure was alcohol consumption, (3) at least three categories of alcohol consumption and a unit of alcohol measurement (g/day) were reported, enabling the evaluation of the dose-response relationship, (4) the outcome was the development of hypertension, and hypertension was defined as a blood pressure $\geq 140 / 90 \mathrm{mmHg}$ and/ or taking anti-hypertensive drugs, (5) data were reported separately for men, (6) the study was performed in community or workplace settings, (7) the included subjects were initially free of hypertension, (8) the included subjects were initially free of cancer and severe comorbidities, and (9) the outcome was reported as relative risks, hazard ratios, or odds ratios with $95 \%$ confidence intervals (CI). We excluded studies according to the following criteria: (1) review articles, letters, conference abstracts, retrospective cohort studies, master's or doctoral dissertations, and meta-analyses, (2) did not report the incidence of hypertension as the outcome, (3) animal studies, (4) hospitalized populations, (5) inappropriate definition of hypertension (based on self-reported hypertension), and (6) the definition of a non-drinker 
was ambiguous (did not distinguish social drinkers and/or ex-drinkers). Three investigators (E.S.S., S.H.I., and M.H.J.) rechecked the eligibility of studies for inclusion in the meta-analysis, and any disagreements were resolved by consensus.

\section{Definition of alcohol consumption categories}

To determine the dose-response relationships, the alcohol consumption level was stratified into five categories: non-drinkers and low (0.01 to $20.0 \mathrm{~g} /$ day), moderate (20.1 to $40.0 \mathrm{~g} /$ day), moderate to high (40.1 to $60.0 \mathrm{~g} /$ day), and high (> 60.0 g/day) dose drinkers. Non-drinkers (lifetime abstainers) were allocated to a reference group. To analyse the effects of the different alcohol dosages, the different forms of alcohol consumption were converted to a daily average alcohol consumption (g/day) based on the type of alcohol and the size of a standard drink in the study's country of origin. The midpoint of the consumption ranges was assigned to one of four alcohol categories. When the upper limit of a category was open-ended, we assigned $75 \%$ of the previous interval added to the lower limit of that category.

\section{Data extraction and quality assessment}

Two independent authors (E.S.S. and S.H.I.) extracted data from the selected studies using a data collection form. To minimize problems with the data collection form, we undertook a pilot test and revised the data collection form as needed. We extracted information on the study design, study setting, follow-up duration, age at baseline, number of participants, reference group, comparison by level of alcohol consumption, and controlled variables. Information used in the meta-analysis included odds ratios, relative risks, hazard ratios, $95 \% \mathrm{CI}$, total number of participants, and total number of events. Exposure (alcohol)-related information was a measurement unit (g/day), criteria and/or classification of the level of alcohol consumption and data collection method.

We used the Newcastle-Ottawa Scale (NOS) to assess the methodological quality of the included studies [22]. The star system of the NOS consisted of three categories: (1) selection (o to 4 points), (2) comparability (o to 2 points), and (3) outcome (o to 3 points). The scale ranged from o to 9 , and we considered higher scores of 7 to 9 as high quality (Supplementary Fig. 1 and Supplementary Table 2).

\section{Statistical analysis}

We expressed the effect size as relative risks with $95 \%$ CI. The pooled relative risk estimates were calculated in each alcohol category. To normalize the data, we took the natural logarithms of the relative risk estimates for each level of alcohol consumption from the individual studies and used the natural logarithms of the reported 95\% confidence interval to calculate the standard errors of the $\log$ relative risk estimates. In cases of missing data, we contacted the corresponding author to acquire the appropriate data for the meta-analysis. We imputed the missing statistics, such as $95 \%$ confidence of intervals, after carefully determining the statistics ( $p$ value) that allowed the calculation. All pooled outcomes were determined using a random-effect model to provide more conservative results than using a fixed-effect model, as suggested by DerSimonian and Laird [23].

The heterogeneity among studies was estimated by the Cochran Q test $(p<0.1$ was set to be statistically significant heterogeneity) and the $I^{2}$ statistic [24]. We considered studies with an $I^{2}>60 \%$ as having substantial heterogeneity. To characterize the region-specific incidence of hypertension according to the level of alcohol consumption, we conducted an analysis stratified by region (Asian vs. Western). We undertook a sensitivity analysis to explore the effects by removing studies with possible sources of heterogeneity one-by-one and recalculating the pooled relative risks for the remaining studies. In cases of heterogeneity $\left(I^{2}>60 \%\right)$ after the sensitivity analysis, a subsequent subgroup analysis according to age was performed.

Publication bias was visually assessed by the asymmetry of the funnel plot [25] and quantitatively assessed by the Egger test $(p<0.1$ was considered statistically significant) [26]. All meta-analyses were conducted using RevMan software version 5.3 (The Nordic Cochrane Center, The Cochrane Collaboration, Copenhagen, Denmark; 2014). R version 3.5 was used to perform the Egger test.

\section{RESULTS}

\section{Study characteristics}

Of 13,578 identified articles, 2,517 duplicated records were excluded. After screening the titles and abstracts, 


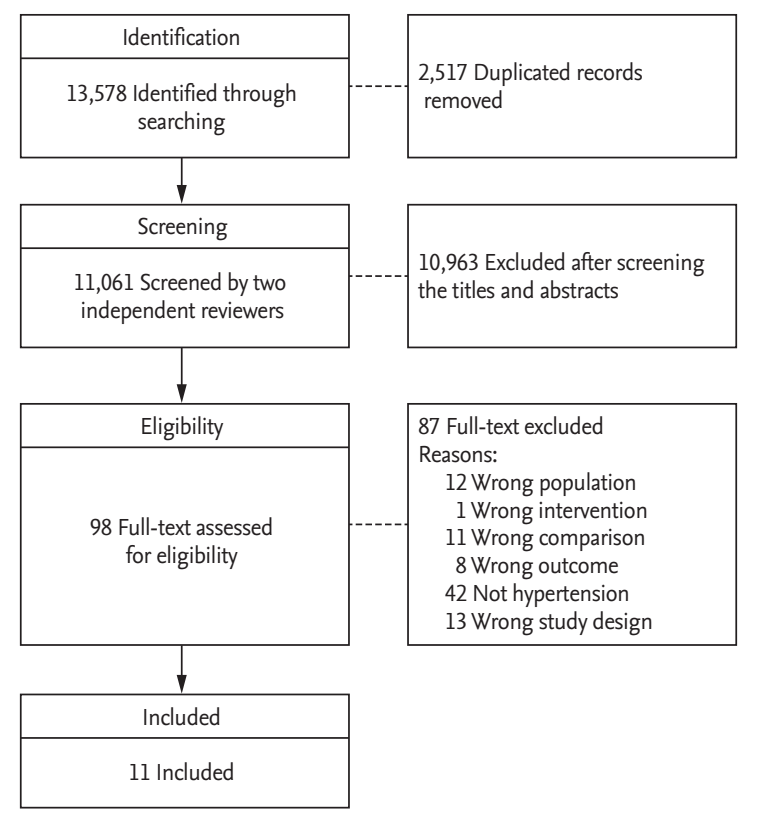

Figure 1. Preferred reporting items for systematic reviews and meta-analyses flow chart for the study selection.

98 were eligible for review of the full text. After full-text reviews, the final meta-analysis included 11 articles. The detailed reasons for exclusion of some studies that included previous meta-analyses are provided in Supplementary Table 3 [27-31]. A flow chart of the study selection is presented in Fig. 1.

The characteristics of the included studies are provided in Table $1[6-8,13,14,17,32-36]$. Seven studies were conducted in Asian countries and four in Western countries. The duration of the follow-up ranged from 3 to 20 years. Among 11 studies, 10 were prospective cohort studies, and one was a case-control study. The included studies had a high quality, as indicated by the nine-star NOS score, and the mean NOS score was 7.5 (ranging from 6 to 9).

\section{Dose-response relationship between the different alcohol consumption categories and the incidence of hypertension: region-specific effect}

We explored the dose-response relationship between alcohol consumption and the incidence of hypertension in each region (Figs. 2 and 3). Among the Asian populations, a significantly elevated risk was observed even in the low alcohol consumption group, which increased in a dose-dependent manner (pooled relative risks [95\% CI] were 1.25 [1.13 to 1.38 ], 1.48 [1.27 to 1.72 ], 1.75 [1.43 to 2.15 , and 1.78 [1.51 to 2.09$]$ for the low, moderate, moderate to high, and high alcohol consumption categories, respectively). Among the Western populations, a similar pattern of increased incidence of hypertension ( $p$ for subgroup difference $>0.1$ ) according to the amount of alcohol consumption was found; however, a significantly elevated risk was evident only in the high-dose group. Specifically, the pooled relative risks (95\% CI) were 1.22 (0.85 to 1.74), 1.57 (0.90 to 2.75), 1.47 (0.44 to 4.91 ), and 1.49 (1.02 to 2.18) for low, moderate, moderate to high, and high doses of alcohol consumption.

\section{Sensitivity and subgroup analysis}

We further performed a sensitivity analysis by removing each individual study with potential sources of heterogeneity. In general, this process did not affect the dose-response relationships. The results of the sensitivity analysis are provided in Table 2 . We further performed a subgroup analysis according to age for the Asian population (Supplementary Table 4). Generally, a similar graded dose-response relationship was demonstrated regardless of age group ( $p$ for interaction by age $>$ o.1), with a tendency of a steeper slope of the association between alcohol consumption and incidence of hypertension in younger men.

\section{Publication bias}

We did not detect any significant publication bias by visual inspection of the funnel plots (Supplementary Figs. 2-5) or by Egger's test (all $p>0.1)$.

\section{DISCUSSION}

The main finding of the current meta-analysis was that there was a graded, dose-dependent increase in risk of hypertension according to alcohol consumption in both Asian and Western men. The risk began at low-dose alcohol consumption (<20.0 g/day of alcohol), which is lower than the generally recommended level of consumption ( 2 units/day $=20-28 \mathrm{~g} /$ day) according to most current guidelines. Furthermore, the elevated risk was more evident in the Asian population. Our results provide additional evidence for avoidance of even a low dose of alcohol consumption to prevent the risk of incident 


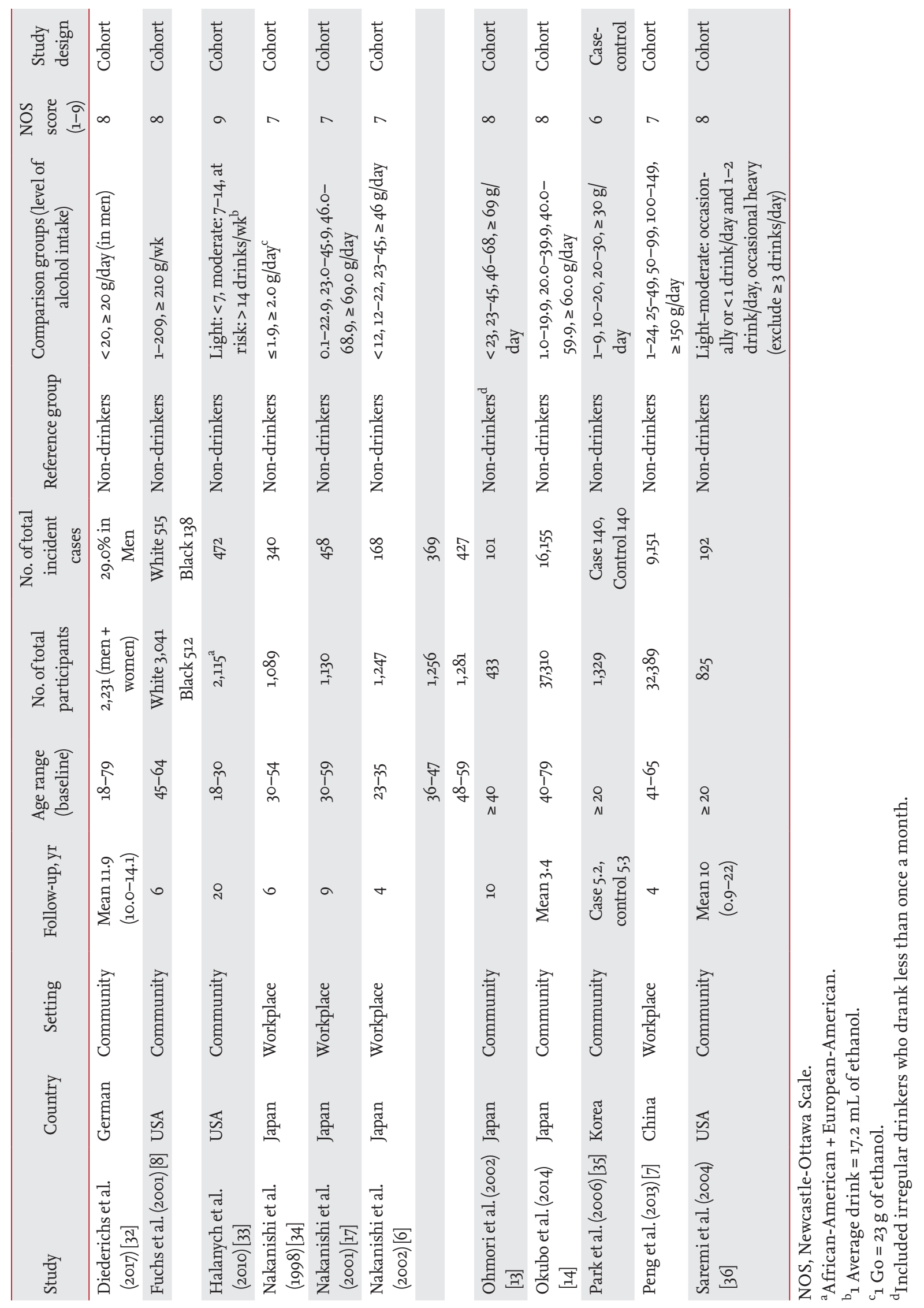




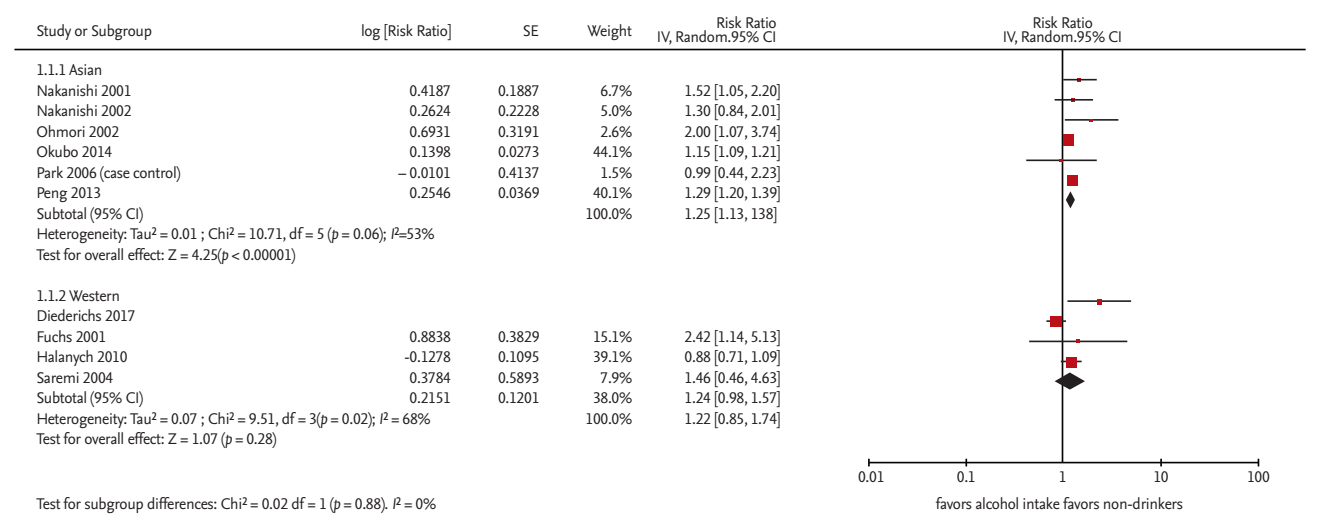

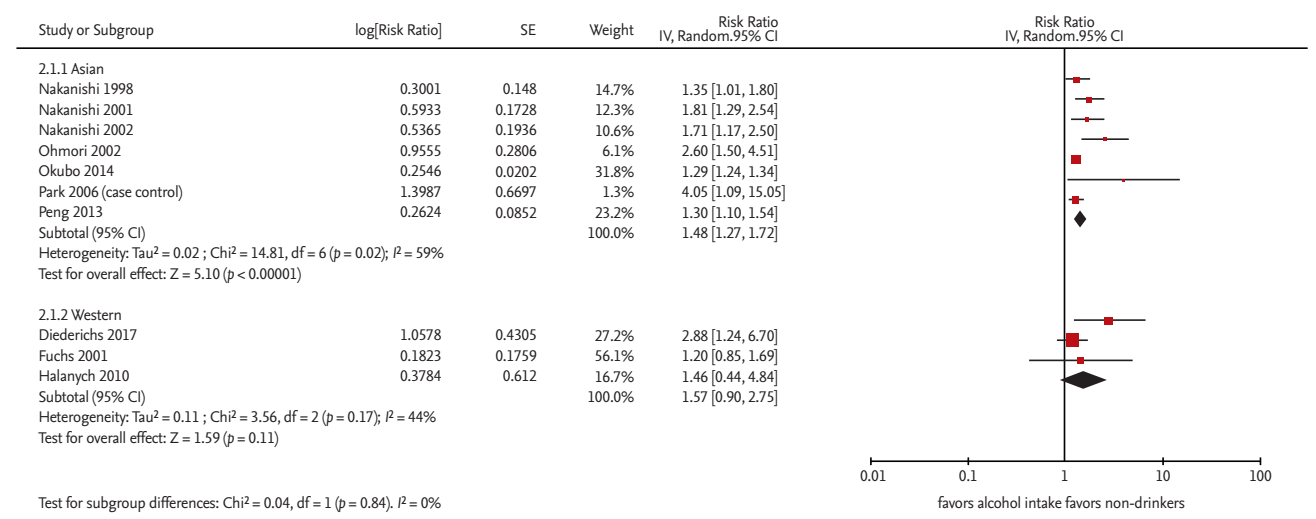

B

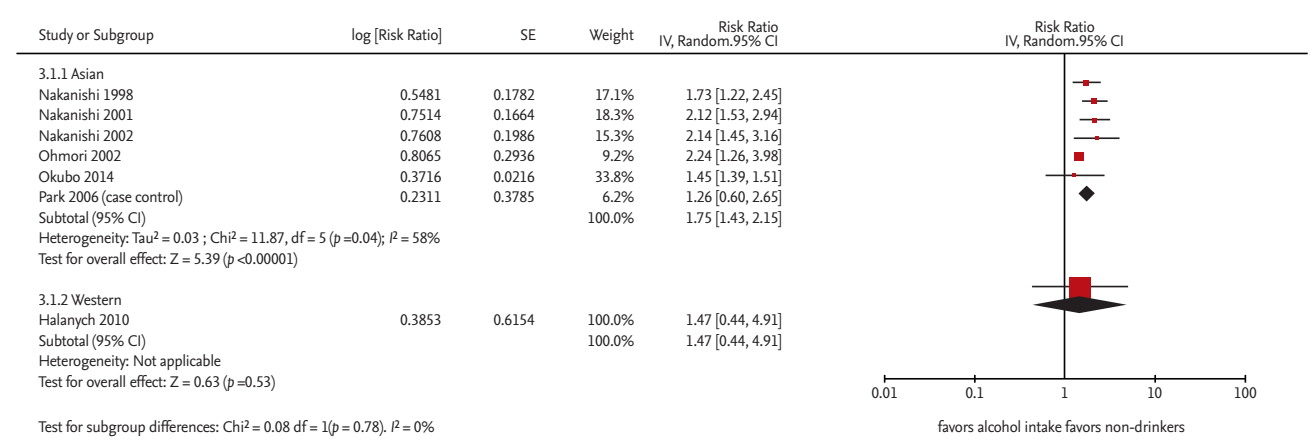

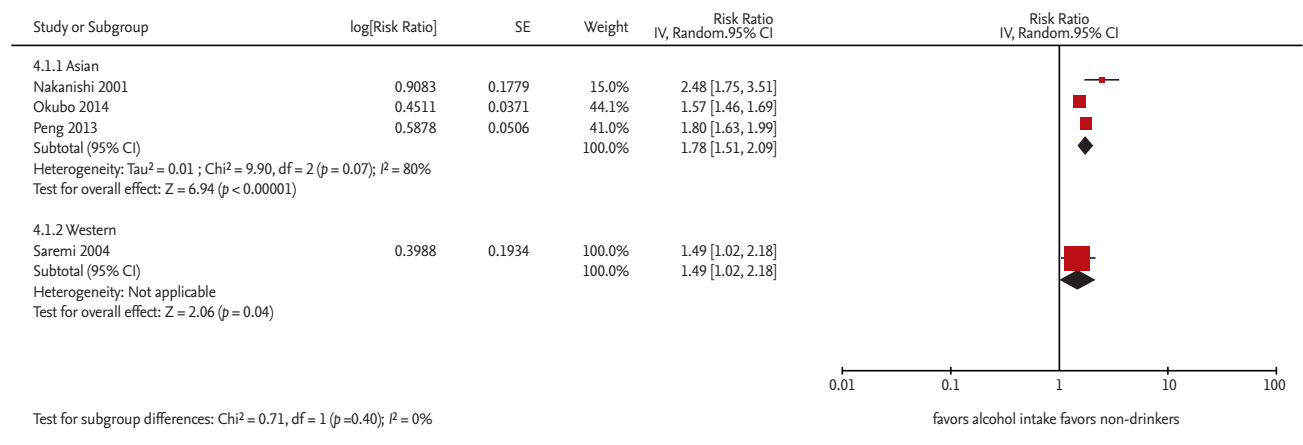

Figure 2. Risk of developing hypertension in each alcohol consumption category, stratified by region. Forest plots for the effect of alcohol dose on hypertension incidence in men: (A) low (o.01 to $20.0 \mathrm{~g} / \mathrm{day}$ ), (B) moderate (20.1 to 40.0 g/day), (C) moderate to high (40.1 to 60.0 g/day), and (D) high (> 60.0 g/day) doses of alcohol consumption. Each plot is stratified by region (Asian and Western). IV, inverse variance; SE, standard error; CI, confidence interval. 
hypertension, particularly in Asian men.

Recent guidelines for hypertension have emphasized the importance of lifestyle modification $[3,4]$. However, controversy still exists regarding low-dose alcohol consumption. Most guidelines permit low-dose alcohol consumption and only limited moderate or greater doses of alcohol consumption [3,4], except for Japanese guidelines [37]. Furthermore, there are heterogeneities in the literature regarding the association between the dose of alcohol consumption and the incidence of hy-

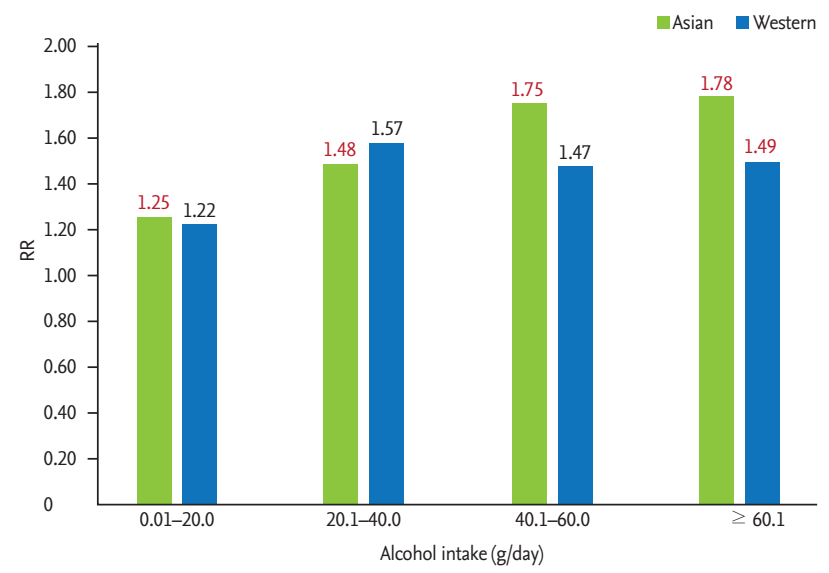

Figure 3. Summary of the dose-response relationship for alcohol consumption and incidence of hypertension among Asian and Western men. RR, relative risk. pertension. In this contemporary meta-analysis, we demonstrated that even low-dose alcohol can elevate the risk for the development of future hypertension, and it was especially evident in Asian men. There was no threshold level below which alcohol consumption exerted a beneficial effect on the development of hypertension both in Asian and Western men. Our data, along with a recent meta-analysis [21], could serve as additional evidence in establishing the recommendations in each region.

There have been limited studies regarding the region- or ethnic-specific effects of alcohol dose on the incidence of hypertension. Fuchs et al. [8] previously reported that the pattern of the dose-response relationship differs according to ethnicity in the Atherosclerosis Risk in Communities cohort. Black men had a significantly increased risk of developing hypertension even at low to moderate alcohol consumption, whereas the risk in other groups (white men and women) did not increase with low-dose consumption [8]. A previous meta-analysis showed that Asian men corresponded to a steeper slope for the association with hypertension risk than non-Asian men at a similar alcohol dosage [19]. In the current analysis, although there was no significant group difference between Asian and Western countries, Asian men exhibited a statistically significant risk el-

Table 2. Hypertension incidence compared to non-drinkers by the level of alcohol consumption

\begin{tabular}{|c|c|c|c|c|c|}
\hline $\begin{array}{l}\text { Alcohol consumption, } \\
\text { g/day }\end{array}$ & Region & $\begin{array}{l}\text { No. of } \\
\text { studies }\end{array}$ & $\begin{array}{c}I^{2}, \% \\
\text { ( } p \text { for heterogeneity) }\end{array}$ & $\begin{array}{c}\mathrm{RR}^{\mathrm{a}} \\
(95 \% \mathrm{CI})\end{array}$ & $\begin{array}{c}p \text { for subgroup } \\
\text { difference }\end{array}$ \\
\hline \multirow[t]{3}{*}{$0.01-20.0$} & Asian & 6 & $53(0.06)$ & $1.25(1.13-1.38)$ & $0.88\left(0.31^{\mathrm{b}}\right)$ \\
\hline & Western & 4 & $68(0.02)$ & $1.22(0.85-1.74)$ & \\
\hline & & $3^{c}$ & $58(0.09)$ & $1.06(0.79-1.43)^{\mathrm{c}}$ & \\
\hline \multirow[t]{2}{*}{$20.1-40.0$} & Asian & 7 & $59(0.02)$ & $1.48(1.27-1.72)$ & 0.84 \\
\hline & Western & 3 & $44(0.17)$ & $1.57(0.90-2.75)$ & \\
\hline \multirow[t]{2}{*}{$40.1-60.0$} & Asian & 6 & $58(0.04)$ & $1.75(1.43-2.15)$ & 0.78 \\
\hline & Western & 1 & - & $1.47(0.44-4.91)$ & \\
\hline \multirow[t]{3}{*}{$>60.0$} & Asian & 3 & $80(<0.01)$ & $1.78(1.51-2.09)$ & $0.40\left(0.22^{b}\right)$ \\
\hline & & $2^{\mathrm{d}}$ & $67(0.08)$ & $2.02(1.49-2.73)^{\mathrm{d}}$ & \\
\hline & Western & 1 & - & $1.49(1.02-2.18)$ & \\
\hline
\end{tabular}

$\mathrm{RR}$, relative risk; CI, confidence interval.

${ }^{\mathrm{a}}$ Generic inverse variance method, random-effect model.

${ }^{\mathrm{b}}$ Sensitivity analysis.

${ }^{\mathrm{c}}$ Sensitivity analysis, a repeat of the primary analysis without an effect estimate from Diederichs and Neuhauser [32].

${ }^{\mathrm{d}}$ Sensitivity analysis, a repeat of the primary analysis without an effect estimate from Okubo et al. [14]. 
evation for each alcohol dose group, whereas Western men corresponded to a marginal graded risk elevation up to moderate to high-dose alcohol consumption (< $60 \mathrm{~g} /$ day), and a significant risk elevation was only evident for high-dose alcohol consumption (> $60 \mathrm{~g} /$ day). Although the exact mechanism for the region- or ethnic-driven heterogeneity is not clear, different body sizes might play a role. Generally, Asian men are leaner than their Western counterparts. A previous study by Nakanishi et al. [17] showed that, among Japanese men, leaner men are more susceptible to developing hypertension with the same dose of alcohol. A higher blood level of alcohol is expected with a similar dose of alcohol consumption in a leaner individual. In addition, differences in alcohol metabolism based on ethnicity might affect the results. A genetic defect in the alcohol degradation enzyme [12] and differences in the gastric first-pass metabolism of alcohol have been reported in the Japanese population [38]. Other racial differences in the renin-angiotensin-aldosterone system, autonomic nervous system, and nitric oxide bioavailability [39-41], which are all implicated in alcohol-induced hypertension [42], might contribute to the difference between the Asian and Western populations. Furthermore, environmental and/or cultural factors, such as eating habits, the type of alcohol, and salt intake, might vary between Asian and Western populations. A recent study demonstrated that the risk of metabolic syndrome could also be affected by the timing of alcohol consumption and the type of alcohol consumed [43].

More comprehensive and detailed lifestyle counselling, beyond salt restriction, is needed to prevent the risk of hypertension. Assessing the subject's drinking history (the amount and pattern of alcohol consumption) is the first step, and further, individualized counselling should also be provided. The survey results of the physicians' awareness and implementation of lifestyle modifications were amazingly disappointing. Awareness and the implementation of alcohol moderation were lowest among the various lifestyle modifications. Less than half of physicians (even less than $20 \%$ in the worst group) actually advised their patients to moderate their alcohol consumption [5]. Considering the linear dose-response relationship of alcohol on the incidence of hypertension, more attention and proactive counselling, namely restriction of alcohol consumption in Asian men and moderation of alcohol consumption in Western men, is urged.

The present study has several strengths. The definition of a non-drinker pertained exclusively to lifetime abstainers, and we excluded past drinkers and rare drinkers. We reduced the possibility of misclassification originating from self-reported hypertension by limiting the definition of hypertension to a blood pressure of $\geq$ 140/90 $\mathrm{mmHg}$ and/or blood pressure medication use. To identify the effect of low to moderate doses of alcohol on the incidence of hypertension, we meticulously subdivided the alcohol dose categories. To our knowledge, this is the first study to directly evaluate the region-specific influence of alcohol consumption on the incidence of hypertension.

Several limitations also need to be addressed. First, the included studies were all observational studies; thus, we cannot entirely exclude the possibility of unadjusted confounding factors. Second, the amount of alcohol consumed by subjects included in this analysis may be over- or under-estimated due to differences in the types of alcohol, the methods of calculating the amount of alcohol consumption, units of measurement, and recall bias. Third, there might be unadjusted confounding factors, such as socioeconomic, educational, and cultural factors, that were not available in this meta-analysis. Alcohol-induced hypertension is a multi-factorial disease that could be affected by various pathophysiological and environmental factors [42]. Fourth, the tendency to publish only positive results might have affected this meta-analysis. However, we did not find evidence of publication bias in this study.

In summary, in this contemporary meta-analysis that explored the region-specific impact of alcohol on the risk of hypertension, we found that alcohol elevates the risk for future hypertension in a dose-dependent manner, and the risk began at an alcohol consumption level lower than the level recommended in most current guidelines for both Asian and Western men. Furthermore, the elevated risk was more evident in Asian men. Based on this study, it is recommended that Asian men avoid even low-dose alcohol consumption and that Western men avoid high-dose alcohol consumption while limiting their overall alcohol consumption to lower levels. Our findings could serve as additional evidence for developing an appropriate preventive strategy in each region. 


\section{KEY MESSAGE}

1. In this contemporary meta-analysis, we found that an increased risk of developing hypertension began in the low-dose alcohol consumption group (<20.0 g/day of alcohol), even though that dose was lower than the generally recommended level of alcohol consumption in most current guidelines ( 2 units/day $=20-28 \mathrm{~g} /$ day). This increased risk was more evident in Asian men than in Western men.

2. Considering the dose-dependent effect of alcohol consumption on the risk of developing hypertension, it is recommended that Asian men avoid consuming even low doses of alcohol and that Western men avoid consuming high doses of alcohol and limit their overall consumption.

\section{Conflict of interest}

No potential conflict of interest relevant to this article was reported.

\section{Acknowledgments}

This research was supported by a fund from the Korea Centers for Disease Control and Prevention (\#2016ER6401-00). The authors wish to thank Jeonga Kim and Miran Jang (Medical Librarian, The Catholic University of Korea, Bucheon St. Mary's Hospital) for their help in searching databases.

\section{REFERENCES}

1. GBD 2015 Risk Factors Collaborators. Global, regional, and national comparative risk assessment of 79 behavioural, environmental and occupational, and metabolic risks or clusters of risks, 1990-2015: a systematic analysis for the Global Burden of Disease Study 2015. Lancet 2016;388:1659-1724.

2. Roerecke M, Kaczorowski J, Tobe SW, Gmel G, Hasan OSM, Rehm J. The effect of a reduction in alcohol consumption on blood pressure: a systematic review and meta-analysis. Lancet Public Health 2017;2:e108-e12o.

3. Williams B, Mancia G, Spiering W, et al. 2018 Practice guidelines for the management of arterial hypertension of the European Society of Hypertension and the European Society of Cardiology: ESH/ESC Task Force for the Management of Arterial Hypertension. J Hypertens 2018;36:2284-2309.

4. Whelton PK, Carey RM, Aronow WS, et al. 2017 ACC/AHA/ AAPA/ABC/ACPM/AGS/APhA/ASH/ASPC/NMA/PCNA guideline for the prevention, detection, evaluation, and management of high blood pressure in adults: executive summary: a report of the American College of Cardiology/American Heart Association Task Force on clinical practice guidelines. Circulation 2018;138:e426-e483.

5. Bolbrinker J, Zaidi Touis L, Gohlke H, Weisser B, Kreutz R. European guidelines on lifestyle changes for management of hypertension: awareness and implementation of recommendations among German and European physicians. Herz 2018;43:352-358.

6. Nakanishi N, Makino K, Nishina K, Suzuki K, Tatara K. Relationship of light to moderate alcohol consumption and risk of hypertension in Japanese male office workers. Alcohol Clin Exp Res 2002;26:988-994.

7. Peng M, Wu S, Jiang X, Jin C, Zhang W; Kailuan Cardiovascular Survey Group. Long-term alcohol consumption is an independent risk factor of hypertension development in northern China: evidence from Kailuan study. J Hypertens 2013;31:2342-2347.

8. Fuchs FD, Chambless LE, Whelton PK, Nieto FJ, Heiss G. Alcohol consumption and the incidence of hypertension: the Atherosclerosis Risk in Communities Study. Hypertension 2001;37:1242-1250.

9. Klatsky AL, Friedman GD, Siegelaub AB, Gerard MJ. Alcohol consumption and blood pressure. Kaiser-Permanente Multiphasic Health Examination data. N Engl J Med 1977;296:1194-1200.

10. Witteman JC, Willett WC, Stampfer MJ, et al. Relation of moderate alcohol consumption and risk of systemic hypertension in women. Am J Cardiol 1990;65:633-637.

11. Frezza M, di Padova C, Pozzato G, Terpin M, Baraona E, Lieber CS. High blood alcohol levels in women. The role of decreased gastric alcohol dehydrogenase activity and first-pass metabolism. N Engl J Med 1990;322:95-99.

12. Shibuya A, Yasunami M, Yoshida A. Genotype of alcohol dehydrogenase and aldehyde dehydrogenase loci in Japanese alcohol flushers and nonflushers. Hum Genet 1989;82:14-16.

13. Ohmori S, Kiyohara Y, Kato I, et al. Alcohol intake and future incidence of hypertension in a general Japanese 
population: the Hisayama study. Alcohol Clin Exp Res 2002;26:1010-1016.

14. Okubo Y, Sairenchi T, Irie F, et al. Association of alcohol consumption with incident hypertension among middle-aged and older Japanese population: the Ibarakai Prefectural Health Study (IPHS). Hypertension 2014;63:41-47.

15. Rehm J, Mathers C, Popova S, Thavorncharoensap M, Teerawattananon Y, Patra J. Global burden of disease and injury and economic cost attributable to alcohol use and alcohol-use disorders. Lancet 2009;373:2223-2233.

16. Radi S, Lang T, Lauwers-Cances V, et al. One-year hypertension incidence and its predictors in a working population: the IHPAF study. J Hum Hypertens 2004;18:487494.

17. Nakanishi N, Yoshida H, Nakamura K, Suzuki K, Tatara K. Alcohol consumption and risk for hypertension in middle-aged Japanese men. J Hypertens 2001;19:851-855.

18. Klatsky AL, Gunderson E. Alcohol and hypertension: a review. J Am Soc Hypertens 2008;2:307-317.

19. Taylor B, Irving HM, Baliunas D, et al. Alcohol and hypertension: gender differences in dose-response relationships determined through systematic review and meta-analysis. Addiction 2009;104:1981-1990.

20. Briasoulis A, Agarwal V, Messerli FH. Alcohol consumption and the risk of hypertension in men and women: a systematic review and meta-analysis. J Clin Hypertens 2012;14:792-798.

21. Roerecke M, Tobe SW, Kaczorowski J, et al. Sex-specific associations between alcohol consumption and incidence of hypertension: a systematic review and meta-analysis of cohort studies. J Am Heart Assoc 2018;7:eoo8202.

22. Lo CK, Mertz D, Loeb M. Newcastle-Ottawa Scale: comparing reviewers' to authors' assessments. BMC Med Res Methodol 2014;14:45.

23. DerSimonian R, Laird N. Meta-analysis in clinical trials. Control Clin Trials 1986;7:177-188.

24. Higgins JP, Thompson SG, Deeks JJ, Altman DG. Measuring inconsistency in meta-analyses. BMJ 2003;327:557-560.

25. Duval S, Tweedie R. Trim and fill: a simple funnel-plotbased method of testing and adjusting for publication bias in meta-analysis. Biometrics 2000;56:455-463.

26. Egger M, Davey Smith G, Schneider M, Minder C. Bias in meta-analysis detected by a simple, graphical test. BMJ 1997;315:629-634.

27. Sesso HD, Cook NR, Buring JE, Manson JE, Gaziano JM.
Alcohol consumption and the risk of hypertension in women and men. Hypertension 2008;51:1080-1087.

28. Tsuruta M, Adachi H, Hirai Y, Fujiura Y, Imaizumi T. Association between alcohol intake and development of hypertension in Japanese normotensive men: 12-year follow-up study. Am J Hypertens 2000;13(5 Pt 1):482-487.

29. Stranges S, Wu T, Dorn JM, et al. Relationship of alcohol drinking pattern to risk of hypertension: a population-based study. Hypertension 2004;44:813-819.

30. Yamada Y, Ishizaki M, Kido T, et al. Alcohol, high blood pressure, and serum gamma-glutamyl transpeptidase level. Hypertension 1991;18:819-826.

31. Curtis AB, James SA, Strogatz DS, Raghunathan TE, Harlow S. Alcohol consumption and changes in blood pressure among African Americans. The Pitt County Study. Am J Epidemiol 1997;146:727-733.

32. Diederichs C, Neuhauser H. The incidence of hypertension and its risk factors in the German adult population: results from the German National Health Interview and Examination Survey 1998 and the German Health Interview and Examination Survey for Adults 2008-2011. J Hypertens 2017;35:250-258.

33. Halanych JH, Safford MM, Kertesz SG, et al. Alcohol consumption in young adults and incident hypertension: 20-year follow-up from the Coronary Artery Risk Development in Young Adults Study. Am J Epidemiol 2010;171:532-539.

34. Nakanishi N, Nakamura K, Ichikawa S, Suzuki K, Kawashimo $H$, Tatara K. Risk factors for the development of hypertension: a 6-year longitudinal study of middle-aged Japanese men. J Hypertens 1998;16:753-759.

35. Park HA, Kim YS, Sun WS. Risk factors of hypertension among Korean Adults. J Korean Acad Fam Med 2006;27:917-926.

36. Saremi A, Hanson RL, Tulloch-Reid M, Williams DE, Knowler WC. Alcohol consumption predicts hypertension but not diabetes. J Stud Alcohol 2004;65:184-190.

37. Kario K. Key points of the Japanese society of hypertension guidelines for the management of hypertension in 2014. Pulse 2015;3:35-47.

38. Dohmen K, Baraona E, Ishibashi H, et al. Ethnic differences in gastric sigma-alcohol dehydrogenase activity and ethanol first-pass metabolism. Alcohol Clin Exp Res 1996;20:1569-1576.

39. Rifkin DE, Khaki AR, Jenny NS, et al. Association of renin and aldosterone with ethnicity and blood pressure: the 
Multi-Ethnic Study of Atherosclerosis. Am J Hypertens 2014;27:801-810.

40. Wolff PH. Ethnic differences in alcohol sensitivity. Science 1972;175:449-450.

41. Mata-Greenwood E, Chen DB. Racial differences in nitric oxide-dependent vasorelaxation. Reprod Sci 2008;15:9-25.
42. Husain K, Ansari RA, Ferder L. Alcohol-induced hypertension: mechanism and prevention. World J Cardiol 2014;6:245-252.

43. Vieira BA, Luft VC, Schmidt MI, et al. Timing and type of alcohol consumption and the metabolic syndrome: ELSA-Brasil. PLoS One 2016;11:e0163044. 
Supplementary Table 1. EMBASE search strategy

\begin{tabular}{|c|c|c|}
\hline No. & Search query & Results \\
\hline \#1 & ‘alcoholic cardiomyopathy'/exp & 944 \\
\hline$\# 2$ & 'cardiovascular disease'/exp OR cardiovascular NEAR/3 disease* & $3,602,305$ \\
\hline \#3 & myocardial NEAR/3 infarction* OR 'heart attack' OR 'heart attacks' & 239,452 \\
\hline \#4 & 'hypertension'/exp OR hypertens* OR high NEAR/3 'blood pressure' & 837,999 \\
\hline \#5 & 'atrial fibrillation'/exp OR (atrial OR auricular) NEAR/3 fibrillation* & 117,233 \\
\hline \#6 & 'variant angina pectoris'/exp OR vasospastic NEAR/3 angina & 7,973 \\
\hline \#7 & $\begin{array}{l}\text { (ischemic OR ischemia* OR infarction*) NEAR/3 (myocardial OR heart OR cardiac OR cardio- } \\
\text { vasc }^{\star} \text { ) }\end{array}$ & 521,299 \\
\hline \#8 & $\begin{array}{l}\text { coronary NEAR/3 ('artery disease' OR 'artery diseases' OR atheroscleros* OR 'heart disease' } \\
\text { OR 'heart diseases') }\end{array}$ & 275,578 \\
\hline \#9 & (heart OR cardiac OR myocardial) NEAR/3 failure* & 334,296 \\
\hline$\# 10$ & ‘cerebrovascular accident'/exp OR stroke OR strokes & 391,261 \\
\hline \#11 & (cerebrovascular OR ‘brain vascular’ OR cerebrovascular) NEAR/3 (accident* OR infarction*) & 254,471 \\
\hline$\# 12$ & 'brain hemorrhage'/exp OR (brain OR intracranial) NEAR/3 hemorrhage* & 111,601 \\
\hline$\# 13$ & \#2 OR \#3 OR \#4 OR \#5 OR \#6 OR \#7 OR \#8 OR \#9 OR \#10 OR \#11 OR \#12 & $3,780,448$ \\
\hline$\# 14$ & $\begin{array}{l}\text { ‘drinking behavior'/exp OR ‘binge drinking'/exp OR ‘alcohol intoxication'/exp OR 'alcoholism'/ } \\
\text { exp OR ‘alcoholic beverage'/exp }\end{array}$ & 172,293 \\
\hline$\# 15$ & 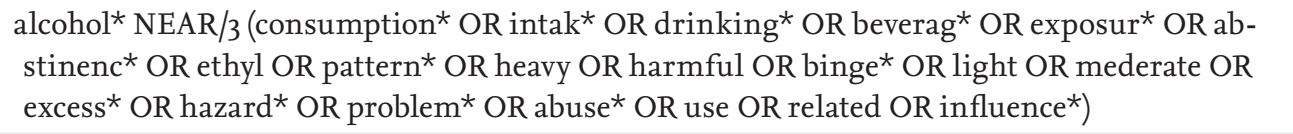 & 205,828 \\
\hline$\# 16$ & $\begin{array}{l}\text { (drink* OR drunk*) NEAR/3 (pattern* OR heavy OR harmful OR binge* OR light OR meder- } \\
\text { ate OR excess* OR hazard* OR problem* OR abuse* OR use OR related OR influence*) }\end{array}$ & 29,747 \\
\hline$\# 17$ & alcoholic OR ethanol & 193,925 \\
\hline \#18 & \#14 OR \#15 OR \#16 OR \#17 & 435,852 \\
\hline$\# 19$ & \#13 AND \#18 & 55,437 \\
\hline$\# 20$ & \#1 OR \#19 & 55,437 \\
\hline \#21 & 'incidence'/exp OR incidenc* & 941,098 \\
\hline \#22 & 'mortality’/exp OR mortalit* OR ‘death rate' OR ‘death rates' & $1,109,574$ \\
\hline \#23 & $\begin{array}{l}\text { 'adverse drug reaction'/exp OR 'adverse effect' OR 'adverse effects' OR 'side effect' OR 'side } \\
\text { effects' }\end{array}$ & $1,286,371$ \\
\hline$\# 24$ & 'dose response'/exp OR 'dose-response' OR ‘dose response' & 415,526 \\
\hline \#25 & \#21 OR \#22 OR \#23 OR \#24 & $3,323,934$ \\
\hline \#26 & \#20 AND \#25 & 18,272 \\
\hline \#27 & $\begin{array}{l}\text { \#26 NOT ([systematic review]/lim OR 'review'/exp OR [meta analysis]/lim OR 'systematic } \\
\text { review'/exp OR 'systematic review (topic)'/exp OR 'meta analysis'/exp OR 'meta analysis' OR } \\
\text { 'meta }\end{array}$ & 14,328 \\
\hline
\end{tabular}


Supplementary Table 2. Star system of the quality assessment used for the New-Castle Ottawa Scale and assigning colors

\begin{tabular}{|c|c|c|c|}
\hline \multicolumn{2}{|c|}{$\begin{array}{l}\text { Star system of Newcastle-Ottawa Scale } \\
\text { (cohort study) }\end{array}$} & \multicolumn{2}{|l|}{ Color assigned } \\
\hline \multirow[t]{6}{*}{ Selection } & \multirow{3}{*}{$\begin{array}{l}\text { 1) Representativeness of the } \\
\text { exposed cohort }\end{array}$} & (a) Truly representative of the average & $\mathrm{a}$ or $\mathrm{b}$ \\
\hline & & & c \\
\hline & & $\begin{array}{l}\text { (describe) in the community } \\
\text { (b) Somewhat } \\
\text { representative of the } \\
\text { average } \\
\text { community } \\
\text { (c) Selected group of users, e.g., nurses, volunteers } \\
\text { (d) No description of the derivation of the cohort }\end{array}$ & $\mathrm{d}$ \\
\hline & $\begin{array}{l}\text { 2) Selection of the nonexposed } \\
\text { cohort }\end{array}$ & $\begin{array}{l}\text { (a) Drawn from the same community as the exposed cohort } \\
\text { (b) Drawn from a different source } \\
\text { (c) No description of the derivation of the nonexposed cohort }\end{array}$ & $\frac{\mathrm{a}}{\mathrm{b}}$ \\
\hline & 3) Ascertainment of exposure & $\begin{array}{l}\text { (a) Secure record (e.g., surgical records) } \\
\text { (b) Structured interview } \\
\text { (c) Written self report } \\
\text { (d) No description }\end{array}$ & $\begin{array}{l}\mathrm{a} \text { or b } \\
\mathrm{c} \\
\mathrm{d}\end{array}$ \\
\hline & $\begin{array}{l}\text { 4) Demonstration that outcome of } \\
\text { interest was not present at start } \\
\text { of study }\end{array}$ & $\begin{array}{l}\text { (a) Yes } \\
\text { (b) No }\end{array}$ & a \\
\hline \multirow[t]{4}{*}{ Comparability } & \multirow[t]{2}{*}{$\begin{array}{l}\text { 1) Study controls for } \\
\text { the most important factor) }\end{array}$} & $\begin{array}{l}\text { (a) Yes } \\
\text { (b) No }\end{array}$ & a \\
\hline & & & - \\
\hline & \multirow{2}{*}{$\begin{array}{l}\text { 2) Study controls for any } \\
\text { additional factor }\end{array}$} & (a) Yes & a \\
\hline & & (b) No & - \\
\hline \multirow[t]{9}{*}{ Outcome } & \multirow[t]{4}{*}{ 1) Assessment of outcome } & & $\mathrm{a}$ or $\mathrm{b}$ \\
\hline & & (a) Independent blind assessment & c \\
\hline & & (b) Record linkage & d \\
\hline & & $\begin{array}{l}\text { (c) Self report } \\
\text { (d) No description }\end{array}$ & \\
\hline & $\begin{array}{l}\text { 2) Was follow-up long enough for } \\
\text { outcomes to occur }\end{array}$ & $\begin{array}{l}\text { (a) Yes (select an adequate follow-up period for the outcome of } \\
\text { interest) } \\
\text { (b) No }\end{array}$ & $\begin{array}{l}\mathrm{a} \\
- \\
\mathrm{b}\end{array}$ \\
\hline & \multirow[t]{4}{*}{ 3) Adequacy of follow-up of cohorts } & (a) Complete follow-up: all subjects accounted for & $\mathrm{a}$ or $\mathrm{b}$ \\
\hline & & $\begin{array}{l}\text { (b) Subjects lost to follow-up unlikely to introduce bias: small } \\
\text { number lost } \rightarrow 10 \% \text { (select an adequate \%) follow-up, or de- } \\
\text { scription provided of those lost) }\end{array}$ & c \\
\hline & & $\begin{array}{l}\text { (C) Follow-up rate }<30 \% \text { (select an adequate \%) and no descrip- } \\
\text { tion of those lost }\end{array}$ & d \\
\hline & & (d) No statement & \\
\hline
\end{tabular}


Supplementary Table 3. Studies that were excluded from the current meta-analysis

\begin{tabular}{ll}
\hline Study & \multicolumn{1}{c}{ Reasons for exclusion } \\
\hline Sesso et al. (2008) [27] & Wrong reference group: It includes rarely drinkers. \\
$\begin{array}{l}\text { Tsuruta et al. (2000) [28] } \\
\text { Stranges et al. (2004) [29] }\end{array}$ & $\begin{array}{l}\text { Wrong reference group: It includes rarely drinkers (0-3 g/day). } \\
\text { consumption of < 12 drinks during their lifetime or in any 1-year period. }\end{array}$ \\
Yamada et al. (1991) [30] & $\begin{array}{l}\text { Wrong comparison: Two groups, subjects with or without an elevated serum r-glutamyl } \\
\text { transpeptidase, were compared; } \\
\text { Unavailable to assess the effect size in the form of relative risks (or odds ratio/hazard ratio) } \\
\text { and 95\% confidence intervals }\end{array}$ \\
Curtis et al. (1997) [31] & $\begin{array}{l}\text { Wrong outcome: Hypertension was defined as a blood pressure } \geq 160 / 95 \text { mmHg or taking } \\
\text { anti-hypertensive medications. }\end{array}$ \\
\hline
\end{tabular}


Supplementary Table 4. Subgroup analysis of the incidence of hypertension by age group among Asian men

\begin{tabular}{|c|c|c|c|c|c|}
\hline $\begin{array}{l}\text { Alcohol consumption, } \\
\text { g/day }\end{array}$ & Age group, yr & No. of studies & $\begin{array}{c}I^{2}, \%(p \text { for } \\
\text { heterogeneity) }\end{array}$ & $\begin{array}{c}\mathrm{RR}^{\mathrm{a}} \\
(95 \% \mathrm{CI})\end{array}$ & $\begin{array}{c}p \text { for subgroup } \\
\text { difference }\end{array}$ \\
\hline \multirow[t]{2}{*}{$0.01-20.0$} & -35 & 1 & - & $1.28(0.76-2.16)$ & 0.94 \\
\hline & $36+$ & 4 & $44(0.15)$ & $1.31(1.06-1.61)$ & \\
\hline \multirow[t]{3}{*}{$20.1-40.0$} & -35 & 1 & - & $2.08(1.24-3.49)$ & $0.32\left(0.18^{b}\right)$ \\
\hline & $36+$ & 5 & $67(0.02)$ & $1.56(1.26-1.93)$ & \\
\hline & & $4^{c}$ & $49(0.12)$ & $1.43(1.21-1.69)^{b}$ & \\
\hline \multirow[t]{3}{*}{$40.1-60.0$} & -35 & 1 & - & $2.45(1.35-4.45)$ & $0.35\left(0.54^{b}\right)$ \\
\hline & $36+$ & 5 & $66(0.02)$ & $1.81(1.44-2.26)$ & \\
\hline & & $4^{d}$ & o (0.79) & $2.01(1.66-2.44)^{b}$ & \\
\hline
\end{tabular}

RR, relative risk; CI, confidence interval.

${ }^{\mathrm{a}}$ Generic inverse variance method, random effect model.

${ }^{\mathrm{b}}$ Sensitivity analysis.

${ }^{\mathrm{c}}$ Sensitivity analysis, a repeat of the primary analysis without an effect estimate from Ohmori et al. [13].

${ }^{\mathrm{d}}$ Sensitivity analysis, a repeat of the primary analysis without an effect estimate from Okubo et al. [14]. 


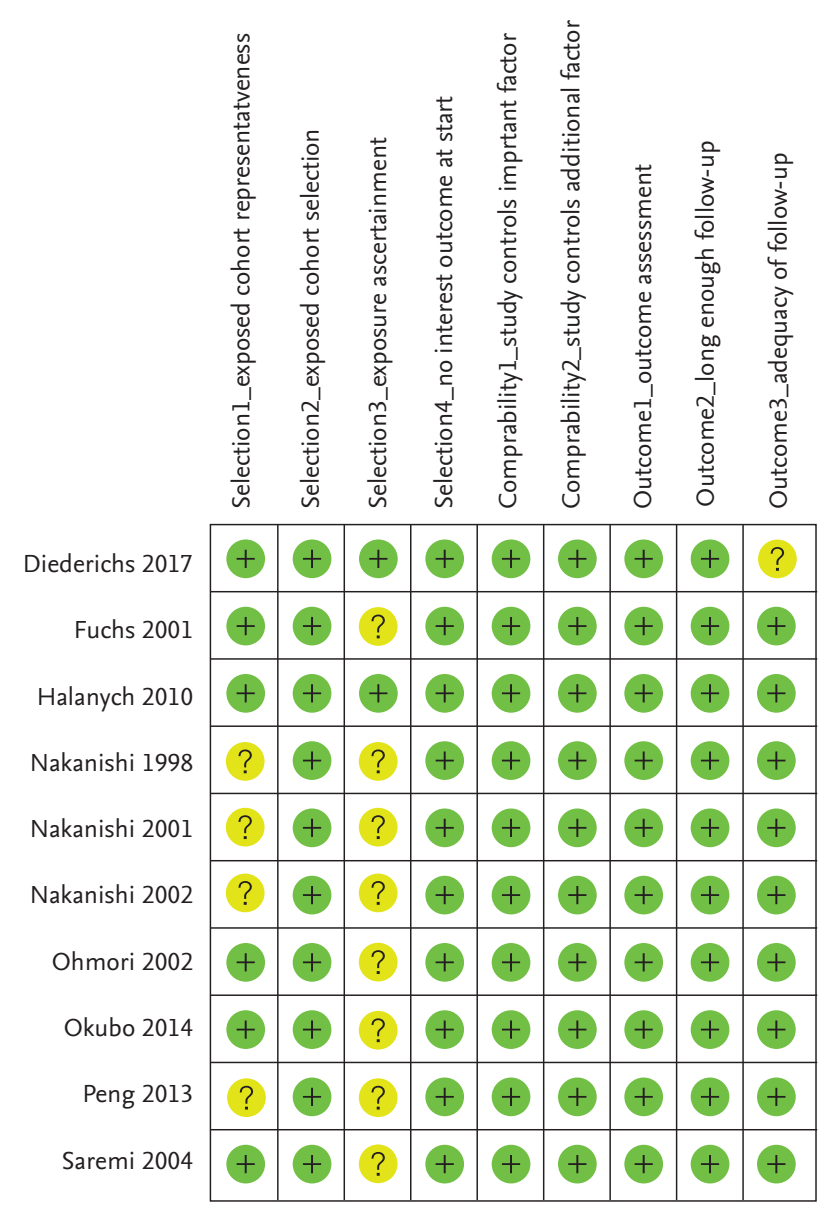

Supplementary Figure 1. Summary of the quality assessment in non-randomized studies: review the authors' judgement about each assessment item for 10 cohort studies. 


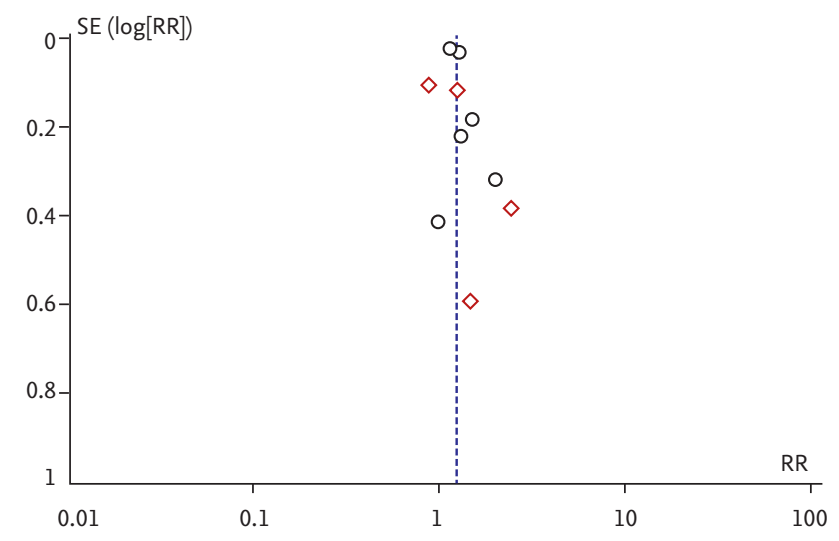

Subgroups

○ Asian (<12 g/day, Nakanishi 2002; 1-9 g/day, Park 2006)

$\diamond$ Western (white, Euro-Am)

Supplementary Figure 2. Funnel plot for the studies regarding low dose alcohol at baseline in men. SE, standard error; $\mathrm{RR}$, relative risk. 


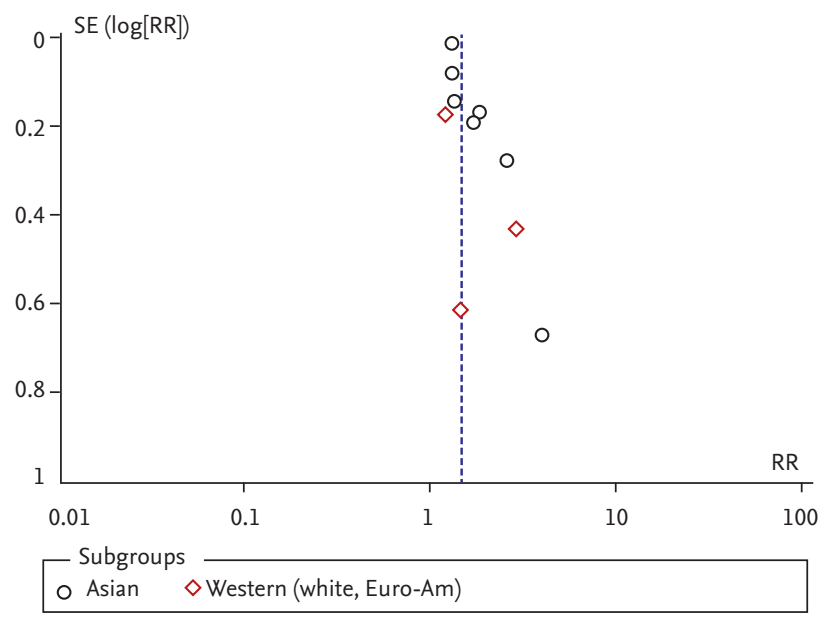

Supplementary Figure 3. Funnel plot for the studies regarding moderate dose alcohol at baseline in men. SE, standard error; RR, relative risk. 


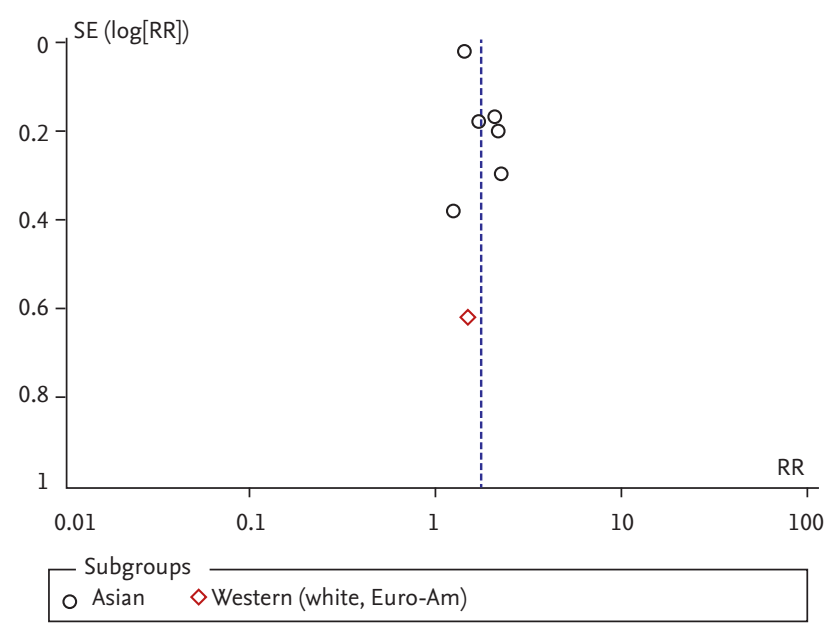

Supplementary Figure 4. Funnel plot for the studies regarding moderate to high dose alcohol at baseline in men. SE, standard error; $\mathrm{RR}$, relative risk. 


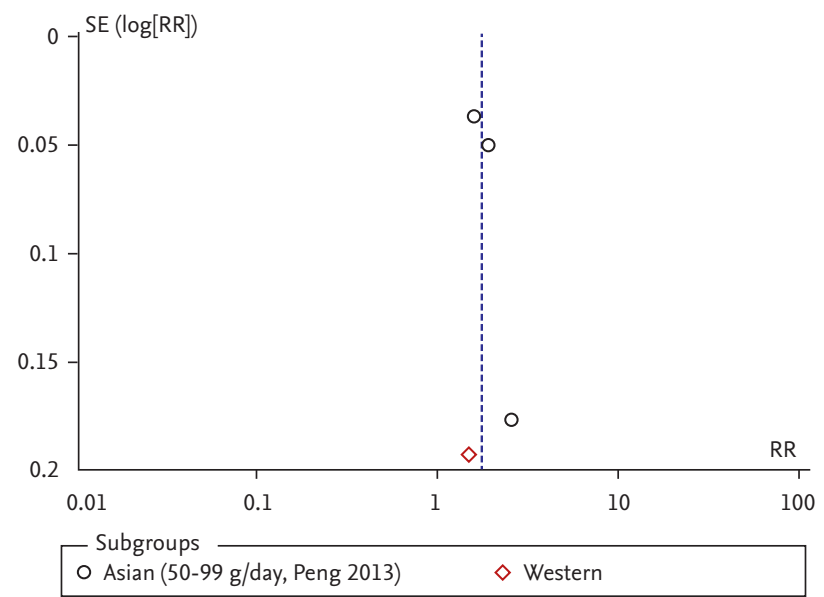

Supplementary Figure 5. Funnel plot for the studies regarding high dose alcohol at baseline in men. SE, standard error; $\mathrm{RR}$, relative risk. 